\title{
Ternary liquid - liquid equilibria for methyl isopropyl ketone + (resorcinol or hydroquinone) + water systems at different temperatures
}

\author{
Yun Chen, Ran Lv, Huimin Wang, Mochen Liao and Libo Li* \\ Key Laboratory of Heat Transfer Enhancement and Energy Conservation of Education \\ Ministry, School of Chemistry and Chemical Engineering, South China University of \\ Technology, Guangzhou, 510640, P.R.China
}

* Correspondence author

Tel.: + 8613902325164

E-mail address: celbli@scut.edu.cn (L.B. Li)

\begin{abstract}
Liquid-liquid equilibrium (LLE) data for the ternary systems, methyl isopropyl ketone $(\mathrm{MIPK})+($ resorcinol, or hydroquinone $)+$ water, were measured at 298.2, 313.2 and $323.2 \mathrm{~K}$ under atmospheric pressure by gas chromatography. The efficiency of MIPK to extract resorcinol or hydroquinone was assessed by the distribution coefficients and separation factors. The LLE data were correlated by the NRTL and UNIQUAC models to yield binary interaction parameters. MIPK was proven to be very promising to extract phenols by comparing its extraction efficiency and physical properties with other extractants used for extracting phenols in industry. The binary interaction parameters of solute pairs, e.g. MIPK-water, calculated from the NRTL or UNIQUAC models were found to agree with corresponding parameters in other LLE systems.
\end{abstract}

Keywords: Liquid-liquid equilibrium; Resorcinol; Hydroquinone; Methyl isopropyl ketone; NRTL; UNIQUAC 


\section{Introduction}

Phenolic compounds (or phenols) include volatile phenols (e.g. phenol, cresols) and non-volatile phenols (e.g. dihydric phenols and trihydric phenols). They are main pollutants in wastewaters produced by a wide range of industries, such as petroleum refineries, petrochemical production, coal conversion, plastic plants, pharmaceuticals firm, and even olive mills $[1,2]$. The concentration of phenols in these industrial effluents can be as high as $10^{4} \mathrm{mg} / \mathrm{kg}$, with that of non-volatile phenols being thousands of $\mathrm{mg} / \mathrm{kg}$ [3-5]. Phenols have been recognized among the most hazardous pollutants, highly toxic even at low concentrations [6] [7], and non-volatile dihydric phenols such as resorcinol and hydroquinone are even more toxic than phenol [8] [9]. Thus, strict regulations have been placed on their concentration in surface water around many countries (e.g. less than $0.5 \mathrm{mg} / \mathrm{kg}$ ) $[5,8]$ and there has been long standing efforts to remove phenols from industrial wastewaters before discharging them to the environment [1]. Furthermore, it would be even better to recover phenols, especially high concentration dihydric phenols, from wastewater, rather than to destroy them, because they are also valuable chemicals with a diverse range of applications: e.g. resorcinol and hydroquinone can be used as photographic developing agent, antioxidants, polymerization inhibitors, drugs or pharmaceuticals [10].

Solvent extraction is an efficient method to recover phenols from wastewaters with various advantages [11, 12], such as high-throughput, ease of automatic operation and of scale up [13], high efficiency for handling high concentration phenols [6], avoiding forming phenol-water azeotrope, a problem leading to incomplete separation by other separation methods (e.g. distillation or stripping) [3], providing economic benefits by recovering phenols [14]. Also, it has been implemented in commercial or industrial plants to treat wastewater (after solvent extraction has reduced the total phenol concentration considerably, biological treatments sometimes are performed to further eliminate these pollutants): e.g. the Great Plains Gasification Plant in U.S.A. [2, 15], the Sasol coal to liquids facility in South Africa [2], China Yima coal gasification plant and Harbin Coal-chemicals Inc. in China [2, 4, 14].

Industrial phenolic wastewater treatment by extraction usually consists of three steps $[4$, 14]: solvent extraction, solvent recovery, and solvent stripping. Firstly, the solvent extraction process is carried out in a solvent extraction column where the wastewater has a 
countercurrent contact with the extraction solvent at each stage. In this step, the phenol concentration in the wastewater is reduced to below $400 \mathrm{mg} / \mathrm{L}$, so that the wastewater is suitable for further biochemical treatment. Second, the extracted wastewater (raffinate) from the bottom of the extraction column is sent to a solvent stripping column to recover the extraction solvent, where the extractant concentration in wastewater is reduced to below 50 $\mathrm{mg} / \mathrm{L}$. Third, the extract from the top of extraction column is sent to a solvent recovery (distillation) column, where the extraction solvent is separated from phenols. Extractant recovered from both the stripping column and the distillation column is sent back to the extraction column for re-use.

It is critical to develop an excellent extractant for extracting phenols from industrial wastewater efficiently and economically and a lot of solvent extraction studies have being performed for such purpose $[1,11]$. In these studies, liquid-liquid equilibrium (LLE) data are measured, and distribution coefficients are calculated from them, which are important to evaluate the extraction efficiency when people develop an extractant to treat phenolic wastewater [11]. Also, the LLE data are correlated with thermodynamic models to yield binary interaction parameters $[16,17]$, which can be used to design or simulate extraction processes in the industrial. The solvent extraction of phenol has been studied thoroughly, and plenty of LLE data have been measured for a wide range of solvents, such as methyl butyl ketone (MBK) [18, 19], methyl isobutyl ketone (MIBK) [20], diisopropyl ether (DIPE) [1, 3], 2-methoxy-2-methylpropane (MTBE) [21], octane [22], 1-octanol [23, 24], Triphenyl phosphate (TPP) and even oleic acid (OA) [25]. Some of these solvents have been used in commercial plants to treat phenolic wastewater $[1,2]$. However, the extraction studies of non-volatile dihydric phenols (e.g. resorcinol and hydroquinone) are very scarce yet, despite that, they are more toxic $[8,9]$, extremely difficult to be biologically degraded $[3,14]$, and usually are highly concentrated (e.g. the concentration is thousands of $\mathrm{mg} / \mathrm{kg}$, comparable to that of phenol) $[2,3]$. In fact, current extractants for industrial phenolic wastewater treatment usually have considerable drawbacks in extracting dihydric phenols, and one reason is that, dihydric phenols are much more harder to extract due to their high affinity with water: e.g. the $\log K_{\mathrm{ow}}$ of hydroquinone (0.59) and resorcinol (0.8) are much smaller than that of phenol (1.46) [9]. DIPE has been used in commercial plants to extract phenols [1], but its capacity for 
extracting resorcinol or hydroquinone is very weak, with distribution coefficients as low as 1 [3]. MIBK show higher distribution coefficients [3, 14], but its boiling point is as high as 389.1 K [26], leading to significantly high energy consumption when recovering this solvent after extraction, either by distillation or solvent stripping [4]. Therefore, more attention should be paid to the extraction of dihydric phenols when continuing to develop better solvents or extraction processes to treat phenolic wastewater.

In this work, methyl isopropyl ketone (MIPK) was studied, with the expectation that this solvent would exhibit even higher efficiency to extract dihydric phenols and more desirable physical properties such as boiling point. Thus, the LLE data for the ternary MIPK + (resorcinol or hydroquinone) + water systems were measured at 298.2, 313.2 and $323.2 \mathrm{~K}$ under atmospheric pressure, which have not been reported yet, to the best of our knowledge. These data were correlated with thermodynamic models including nonrandom two-liquid (NRTL) [17] and universal quasi-chemical activity coefficient (UNIQUAC) [16]. The distribution coefficients and physical properties of MIPK were compared in details with other solvents which have been used for commercial treatment of phenolic wastewater, e.g. MIBK [4] and DIPE [1]. The correlation between distribution coefficients and octanol/water partition coefficients were further discussed. The NRTL or UNIQUAC binary interaction parameters of solute pairs, e.g. MIPK-water, resorcinol-water and hydroquinone-water, calculated in this work agree with parameters of corresponding solute pairs in other LLE systems quite well, which could help understand the physical background of these two thermodynamic models.

\section{Experimental}

\subsection{Materials}

The detailed information of chemical reagents used in this work (e.g. MIPK, resorcinol and hydroquinone, etc.) is shown in Table 1. Gas chromatography was used to verify their purity, and no impurity peak was detected for each reagent. Thus these chemical reagents were used without further purification as typical LLE studies do [5, 27]. Deionized water was used throughout this study.

\subsection{Apparatus and procedure}

The experimental LLE data for the ternary systems, MIPK + (resorcinol or hydroquinone) 
+ water were measured at 298.2, 313.2 and $323.2 \mathrm{~K}$ under atmospheric pressure. Certain amounts of MIPK, resorcinol or hydroquinone, and water were loaded into a $100 \mathrm{~mL}$ glass equilibrium cell to form a ternary mixture. The mixture was agitated vigorously for more than 2 hours and then was left to settle for over 20 hours for the system to reach the phase equilibrium. The temperature of the mixture in the cell was kept constant by using a thermostatic bath with a fluctuation of $0.1 \mathrm{~K}$.

When the phase equilibrium of the as-prepared ternary mixture was reached, and the mixture was split into two distinct layers, the sample of each layer was collected with a syringe, and was analyzed by a gas chromatography (GC 6820, Agilent Technologies) equipped with a DB-5MS capillary column $(30 \mathrm{~m} \times 0.32 \mathrm{~mm} \times 0.25 \mu \mathrm{m})$ and a flame ionization detector (FID). Nitrogen was used as carrier gas with a flow rate of $30 \mathrm{~cm}^{3} \cdot \mathrm{min}^{-1}$. The temperature of the GC oven was controlled as follows: the initial temperature of the column was kept at $313.2 \mathrm{~K}$ for $2 \mathrm{~min}$, increased at a rate of $30 \mathrm{~K}$ per minute to $433.2 \mathrm{~K}$ and then was kept at $433.2 \mathrm{~K}$ for $2 \mathrm{~min}$. The temperatures of the injector and detector were kept at 523.2 and $543.2 \mathrm{~K}$, respectively. The composition of the sample was determined by an internal standard method, where $n$-butyl acetate was used as an internal standard for MIPK and $n$-octanol for resorcinol or hydroquinone. The mass fraction of water was calculated by deducting those of MIPK and resorcinol (or hydroquinone) from 1 (please note that, the total mass fraction of resorcinol or hydroquinone, water and MIPK is exactly 1 for the ternary systems). The samples and internal standards were weighed by an analytical balance (Shimadzu AUW220D) with an accuracy of $0.0001 \mathrm{~g}$. Each sample was measured for at least three times, with a standard deviation of less than $0.2 \%$, thus the average value was reported in this work.

\section{Results and discussion}

\subsection{Experimental LLE data}

The experimental LLE data (in mass fraction) for ternary systems, (MIPK + resorcinol + water) and (MIPK + hydroquinone + water), at $298.2 \mathrm{~K}, 313.2 \mathrm{~K}$ and $323.2 \mathrm{~K}$ under atmospheric pressure, are listed in Tables 2 and 3, respectively. The corresponding triangular phase diagrams are shown in Figs. 1 and 2. 


\section{2 distribution coefficients and separation factors}

To evaluate the capacity of MIPK to recover resorcinol or hydroquinone from aqueous solution, the distribution coefficients $(D)$ and separation factors $(S)$ were calculated with following formula, respectively:

$$
\begin{aligned}
& D=\frac{w_{2}{ }^{\mathrm{II}}}{w_{2}{ }^{\mathrm{I}}} \\
& S=\frac{\left(w_{2} / w_{3}\right)^{\mathrm{II}}}{\left(w_{2} / w_{3}\right)^{\mathrm{I}}}
\end{aligned}
$$

where superscripts I and II refer to the aqueous phase and the organic phase, respectively, $w_{2}$ is the mass fraction of resorcinol or hydroquinone, and $w_{3}$ is that of water. The distribution coefficients and separation factors are shown in Tables 2 and 3. According to these tables, the distribution coefficients of hydroquinone in MIPK are much higher than those MIBK [5] or DIPE [3] (Fig. S1 in the supplementary material). The distribution coefficients of resorcinol in MIPK are close to MIBK [27], but significantly higher than DIPE [3] (Fig. S2 in the supplementary material). These indicate MIPK extracts polyhydric phenols such as hydroquinone or resorcinol very efficiently. The distribution coefficients of phenol in MIPK are also considerably higher than those in MIBK or DIPE according to our ongoing study (unpublished data). In addition, the distribution coefficients of hydroquinone in MIPK, e.g. at 298.2 K, are lower than those of resorcinol (see Tables 2 and 3, and Fig. S1 and S2). This indicates hydroquinone is more difficult to extract by MIPK than resorcinol, which is expected since the $\log K_{\text {ow }}$ of hydroquinone is smaller, indicating greater water affinity. Similar tendency was observed when extracting phenols with DIPE or MIBK: e.g. the distribution coefficients of hydroquinone, resorcinol, catechol and phenol in DIPE or MIBK at $298 \mathrm{~K}$ are $(1.03,2.06,4.86$ and 36.5$)$ or $(9.92,17.9,18.7$ and 100) respectively [3], agreeing with the order of their $\log K_{\text {ow }}: 0.59,0.8,1.0$ and 1.46 [9]. Furthermore, Tables 2 and 3 show that, distribution coefficients and separation factors decrease as the concentration or temperature increases in the studied range. Such dependence of distribution coefficients (or separation factors) on concentration or temperature has been observed in various of other (extractant + phenols + water) ternary systems $[18,28]$. This indicates that, it is necessary to screen possible extractants more carefully, and further strengthen the advantages of MIPK 
over other solvents, when the extract process is designed to perform at elevated temperatures [29] or to treat streams with high phenols concentration [1, 2]. MIPK's boiling point (367.4 K) is considerably lower than MIBK (389.1 K) [26], which indicates this solvent could be recovered after extraction quite easily either by distillation or stripping. Nevertheless, MIPK's boiling point is much higher than DIPE (341.7 K) or MTBE (328.5 K) [26], thus an MIPK based extraction process could be performed at higher temperature to avoid clogging the extraction installation when treating wastewaters with high concentration of paraffin [29]. Thus, MIPK should be a very promising extractant to recover phenols, especially dihydric phenols, from wastewater due to all these advantages.

\subsection{Data Correlation.}

The experimental LLE data for the ternary systems, MIPK + (resorcinol or hydroquinone) + water, at 298.2, 313.2 and $323.2 \mathrm{~K}$, were converted from mass fraction to mole fraction, and then were correlated using the NRTL [17] and UNIQUAC [16] models to yield binary interaction parameters. The UNIQUAC structural parameter $r$ (taken from reference [30]) for MIPK, resorcinol (or hydroquinone), and water are 3.9215, 3.9156 and 0.9200, respectively, while parameter $q$ [30] for these components are 3.4120, 3.0080 and 1.4000, respectively. The binary interaction parameters in the NRTL and UNIQUAC models for our studied systems were calculated by minimizing the objective function (OF):

$$
\mathrm{OF}=\sum_{i=1}^{3} \sum_{j=1}^{2} \sum_{k=1}^{n}\left(x_{i j k}^{\mathrm{exp}}-x_{i j k}^{\mathrm{cal}}\right)^{2}
$$

where subscripts $i, j$ and $k$ refer to the components, the phases and the tie-lines, respectively, $n$ is the number of tie-lines, $x^{\exp }$ is the experimental mole fraction, and $x^{\text {cal }}$ is the calculated mole fraction. The agreement between experimental data and calculated results from the NRTL or UNIQUAC models was assessed by the root-mean-square-deviation (RMSD), defined as:

$\mathrm{RMSD}=\left[\frac{\sum_{i=1}^{3} \sum_{j=1}^{2} \sum_{k=1}^{n}\left(w_{i j k}^{\mathrm{exp}}-w_{i j k}^{\mathrm{cal}}\right)^{2}}{6 n}\right]^{1 / 2}$

The RMSD values for the NRTL and UNIQUAC models are shown in Table 4. These 
RMSD values are very small, which further verifies that, both NRTL and UNIQUAC models describe the phase behavior of ternary systems in this work quite accurately. Table 4 also list binary interaction parameters calculated from the NRTL and UNIQUAC models, which could be used to design or simulate the extraction process of resorcinol or hydroquinone. Very interestingly, the interaction parameters of MIPK-water, $b_{13}$ and $b_{31}$, in the ternary system, MIPK (1) + resorcinol (2) + water (3), are quite close to those in MIPK (1) + hydroquinone (2) + water (3) in the studied temperature range (Table 4 and Fig. S3 in the supplementary material). Such agreement is also observed for the binary pair of MIBK-water in ternary systems, MIBK (1) + (resorcinol or hydroquinone) (2) + water (3) (Fig. S3) [5, 27]. The interaction parameters of (resorcinol or hydroquinone)-water, $b_{23}$ and $b_{32}$ (Table 4), also agree roughly with corresponding parameters in the ternary MIBK (1) + (resorcinol or hydroquinone) (2) + water (3) systems (Table S1 in the supplementary material). Such agreement is observed in both NRTL and UNIQUAC models, which would help better understand the physical background of these two models.

The calculated mass fractions of resorcinol or hydroquinone in both organic and aqueous phases are shown in Figs. S4 and S5 in the supplementary material, with comparison to experimental data. All these figures show the calculated results are in good agreement with experimental data, which further confirms both NRTL and UNIQUAC models are suitable for simulating the process of extracting resorcinol or hydroquinone with MIPK.

\section{Conclusion}

The LLE data for the ternary systems, MIPK + (resorcinol or hydroquinone $)+$ water, were measured at 298.2, 313.2 and 323.2K under atmospheric pressure. The experimental LLE data were correlated by the NRTL and UNIQUAC models, which both described the phase behavior of the studied systems very accurately and yielded relevant binary interaction parameters among MIPK, water, and resorcinol or hydroquinone. The NRTL or UNIQUAC binary interaction parameters of the ternary MIPK + (resorcinol or hydroquinone) + water systems were compared with parameters of other LLE systems to help better understand these two thermodynamic models. The distribution coefficients of phenolic compounds in a solvent, D, were found to correlate with their $\log K_{\text {ow }}$ MIPK was proven to be a very promising 
extractant to recover non-volatile phenols, such as resorcinol or hydroquinone, from wastewater, since it show considerably better extraction performance and physical properties than other extractants which have been used for similar purpose in industry (e.g. MIBK and DIPE). The experimental LLE data, calculated binary interaction parameters and the correlation between $\mathrm{D}$ and $\log K_{\text {ow }}$ discussed in this work should be useful for designing or optimizing the extraction process of phenolic compounds in industry, especially in the field of wastewater treatment.

\section{Acknowledgments}

Financial support from Project of the Science \& Technology New Star of Pearl River in Guangzhou (2011J2200056), the Fundamental Research Funds for the Central Universities, SCUT (2014ZZ0057 and 2015ZM046), Guangdong Science Foundation (2014A030310260) and National Science Foundation of China (20906028 and 21506066) are gratefully acknowledged.

\section{References}

[1] G. Busca, S. Berardinelli, C. Resini, L. Arrighi, Technologies for the removal of phenol from fluid streams: A short review of recent developments, Journal of hazardous materials, 160 (2008) 265-288.

[2] Q.H. Ji, S. Tabassum, S. Hena, C.G. Silva, G.X. Yu, Z.J. Zhang, A review on the coal gasification wastewater treatment technologies: past, present and future outlook, Journal of Cleaner Production, (2016) In Press, DOI:10.1016/j.jclepro.2016.02.147.

[3] D.C. Greminge, G.P. Burns, S. Lynn, D.N. Hanson, C.J. King, Solvent extraction of phenols from water, Ind. Eng. Chem. Process Des. Dev., 21 (1982) 51-54.

[4] C.F. Yang, S.Y. Yang, Y. Qian, J.W. Guo, Y. Chen, Simulation and Operation Cost Estimate for Phenol Extraction and Solvent Recovery Process of Coal-Gasification Wastewater, Ind Eng Chem Res, 52 (2013) 12108-12115.

[5] C.F. Yang, Y.B. Jiang, L.J. Zhang, Y. Qian, Liquid-liquid equilibria for the ternary system methyl isobutyl ketone plus water plus hydroquinone, J Chem Eng Data, 51 (2006) 2107-2109. 
[6] H. Jiang, Y. Fang, Y. Fu, Q.X. Guo, Studies on the extraction of phenol in wastewater, Journal of hazardous materials, 101 (2003) 179-190.

[7] M. Teresa, A. Reis, O.M.F. de Freitas, M. Rosinda, C. Ismael, J.M.R. Carvalho, Recovery of phenol from aqueous solutions using liquid membranes with Cyanex 923, J Membrane Sci, 305 (2007) 313-324

[8] P.C. Singer, F.K. Pfaender, J. Chinchilli, A.F. Maciorowski, L.J. C., R. Goodman, Assessment of Coal Conversion Wastewters: Characterization and Preliminary Biotreatability, U.S. Environmental protection Agency Office of Research and Development, Washington, DC, 1978.

[9] H. Roche, G. Boge, In vivo effects of phenolic compounds on blood parameters of a marine fish (Dicentrarchus labrax), Comp Biochem Phys C, 125 (2000) 345-353.

[10] A. Afkhami, H.A. Khatami, Indirect kinetic-spectrophotometric determination of resorcinol, catechol, and hydroquinone, J Anal Chem+, 56 (2001) 429-432.

[11] V.S. Kislik, Solvent extraction: classical and novel approaches, Elsevier, Oxford, UK, 2012.

[12] F.M.R. Mesquita, R.S. Pinheiro, H.B. de Sant'Ana, R.S. Santiago-Aguiar, Removal of aromatic hydrocarbons from hydrocarbon mixture using glycols at $303.15 \mathrm{~K}$ and $333.15 \mathrm{~K}$ and atmospheric pressure: Experimental and calculated data by NRTL and UNIQUAC models, Fluid Phase Equilibria, 387 (2015) 135-142.

[13] G. Muthuraman, T.T. Teng, C.P. Leh, I. Norli, Extraction and recovery of methylene blue from industrial wastewater using benzoic acid as an extractant, Journal of hazardous materials, 163 (2009) 363-369.

[14] C.F. Yang, Q.A. Yu, L.J. Zhang, J.Z. Feng, Solvent extraction process development and on-site trial-plant for phenol removal from industrial coal-gasification wastewater, Chemical Engineering Journal, 117 (2006) 179-185.

[15] M.D. Mann, W.G. Willson, J.G. Hendrikson, S.L. Winton, Gasifier wastewater treatment: Phase I cooling tower assessment, Environmental Progress \& Sustainable Energy, 4 (1985) 33-39.

[16] D.S. Abrams, J.M. Prausnitz, Statistical Thermodynamics of Liquid Mixtures: A New expression for the Excess Gibbs Energy of Partly or Completely Miscible Systems, AIChE J., 21 (1975) 116-128.

[17] H. Renon, J.M. Prausnitz, Local Compositions in Thermodynamic Excess Functions for Liquid Mixtures, AlChE Journal, 14 (1968) 135-144.

[18] Y. Chen, Z. Wang, L.B. Li, Liquid Liquid Equilibria for Ternary Systems: Methyl Butyl Ketone 
plus Phenol plus Water and Methyl Butyl Ketone plus Hydroquinone + Water at 298.15 K and 323.15 K, J Chem Eng Data, 59 (2014) 2750-2755.

[19] R. Lv, Z. Wang, L.B. Li, Y. Chen, Liquid-liquid equilibria in the ternary systems water plus cresols plus methyl butyl ketone at 298.2 and $313.2 \mathrm{~K}$ : Experimental data and correlation, Fluid Phase Equilibria, 404 (2015) 89-95.

[20] C.F. Yang, Y. Qian, L.J. Zhang, Y.B. Jiang, Measurement and correlation of liquid - liquid equilibrium data for methyl isobutyl ketone - water - phenol ternary system, Journal of Chemical Industry and Engineering (China), 58 (2007) 805-809.

[21] L.J. Luo, D. Liu, L.B. Li, Y. Chen, Phase equilibria of (water plus propionic acid or butyric acid+2-methoxy-2-methylpropane) ternary systems at $298.2 \mathrm{~K}$ and $323.2 \mathrm{~K}$, Fluid Phase Equilibria, 403 (2015) 30-35.

[22] A. Martin, M. Klauck, A. Grenner, R. Meinhardt, D. Martin, J. Schmelzer, Liquid-Liquid(-Liquid) Equilibria in Ternary Systems of Aliphatic Hydrocarbons (Heptane or Octane) plus Phenols plus Water, J Chem Eng Data, 56 (2011) 741-749.

[23] H.G. Gilani, A.G. Gilani, M. Sangashekan, Tie-line data for the aqueous solutions of phenol with organic solvents at $\mathrm{T}=298.2 \mathrm{~K}$, J Chem Thermodyn, 58 (2013) 142-148.

[24] A.G. Gilani, H.G. Gilani, S.L.S. Saadat, Solubility and tie line data for the aqueous solutions of butyric acid with 1-octanol and 2-ethyl-1-hexanol at various temperatures, Fluid Phase Equilibria, 361 (2014) 45-53.

[25] S. Yoon, S.Y. Kwon, C.W. Lee, An efficient and cost-effective solvent extraction for recovery of phenol and its hydroxy derivatives from aqueous medium, Korean J Chem Eng, 28 (2011) 71-78.

[26] N.L. Cheng, Solvents Handbook, third ed., Chemical Industry Press, Beijing, China, 2002.

[27] C.F. Yang, Y. Qian, J.W. Guo, J.R. Chen, J.P. Peng, Liquid-Liquid Equilibria for the Ternary System Methyl Isobutyl Ketone plus m-Benzenediol plus Water, J Chem Eng Data, 59 (2014) 3324-3328.

[28] Y. Lei, Y. Chen, X.X. Li, Y. Qian, S.Y. Yang, C.F. Yang, Liquid-Liquid Equilibria for the Ternary System 2-Methoxy-2-methylpropane plus Phenol plus Water, J Chem Eng Data, 58 (2013) 1874-1878. [29] H.M. Wang, R. Lv, L.B. Li, Y. Chen, Measurement and correlation of liquid-liquid equilibria for the ternary system methyl isobutyl ketone + phenol + water at $(333.15,343.15$ and 353.15$) \mathrm{K}$ under atmospheric pressure, J. Solu. Chem., (2016) In Press, DOI: 10.1007/s10953-016-0472-z. 
[30] T. Magnussen, P. Rasmussen, A. Fredenslund, UNIFAC Introduction Parameter Table for Prediction of Liquid-Liquid Equilibria, Ind. Eng. Chem. Process Des. Dev., 20 (1981) 331-339. 


\section{Caption of Tables and Figures}

\section{Tables}

\section{Table 1}

Details of materials used in this work.

\section{Table 2}

The experimental LLE data in mass fraction for $\{$ MIPK (1) + resorcinol (2) + water (3) $\}$ system at 298.2, 313.2 and 323.2 K under atmospheric pressure. ${ }^{\mathrm{a}, \mathrm{b}}$

\section{Table 3}

The experimental LLE data in mass fraction for $\{$ MIPK (1) + hydroquinone (2) + water (3) $\}$ system at 298.2, 313.2 and 323.2 K under atmospheric pressure. ${ }^{\mathrm{a}, \mathrm{b}}$

\section{Table 4}

Binary interaction parameters calculated from NRTL and UNIQUAC models for the ternary systems: $\{$ MIPK (1) + (resorcinol or hydroquinone) $(2)+$ water $(3)\}$.

\section{Figures}

Fig. 1. LLE data for the ternary system $\{$ MIPK (1) + resorcinol (2) + water (3) $\}$ at different temperatures: $\mathbf{\square}$, at $298.2 \mathrm{~K} ; \bullet$, at $313.2 \mathrm{~K} ; \boldsymbol{\Delta}$, at $323.2 \mathrm{~K}$.

Fig. 2. LLE data for the ternary system $\{$ MIPK (1) + hydroquinone (2) + water (3) $\}$ at different temperatures: $\mathbf{\square}$, at $298.2 \mathrm{~K} ; \bullet$, at $313.2 \mathrm{~K} ; \boldsymbol{\Delta}$, at $323.2 \mathrm{~K}$. 


\section{Table 1}

Details of materials used in this work.

\begin{tabular}{|c|c|c|c|c|}
\hline Chemical & IUPAC name & CAS & Vendor & Mass purity \\
\hline Methyl isopropyl ketone & 3-Methyl-2-butanone & $563-80-4$ & Xiya reagent & $>0.99$ \\
\hline Resorcinol & 1,3-Benzenediol & $108-46-3$ & Xiya reagent & $>0.99$ \\
\hline Hydroquinone & 1,4-Benzenediol & $123-31-9$ & Xiya reagent & $>0.99$ \\
\hline$n$-Butyl acetate & Butyl acetate & $123-86-4$ & Tianjin Kemiou Chemical Reagent & $>0.99$ \\
\hline$n$-Octanol & 1-Octanol & $111-87-5$ & Tianjin Kemiou Chemical Reagent & $>0.99$ \\
\hline
\end{tabular}




\section{Table 2}

The experimental LLE data in mass fraction for $\{$ MIPK (1) + resorcinol (2) + water (3) $\}$ system at 298.2, 313.2 and 323.2 K under atmospheric pressure. , b $^{\text {b }}$

\begin{tabular}{|c|c|c|c|c|c|c|}
\hline \multirow[t]{2}{*}{$T / \mathrm{K}$} & \multicolumn{2}{|c|}{ Aqueous phase } & \multicolumn{2}{|c|}{ Organic phase } & \multirow[t]{2}{*}{$D$} & \multirow[t]{2}{*}{$S$} \\
\hline & $w_{1}$ & $w_{2}$ & $w_{1}$ & $w_{2}$ & & \\
\hline \multirow[t]{8}{*}{298.2} & 0.0579 & 0.0009 & 0.943 & 0.0196 & 21.78 & 548.1 \\
\hline & 0.0558 & 0.0025 & 0.907 & 0.0485 & 19.40 & 410.5 \\
\hline & 0.0531 & 0.0048 & 0.867 & 0.0799 & 16.65 & 295.3 \\
\hline & 0.0511 & 0.0067 & 0.839 & 0.1025 & 15.30 & 246.4 \\
\hline & 0.0476 & 0.0100 & 0.799 & 0.1334 & 13.34 & 186.0 \\
\hline & 0.0451 & 0.0123 & 0.774 & 0.1524 & 12.39 & 158.7 \\
\hline & 0.0417 & 0.0155 & 0.747 & 0.1755 & 11.32 & 137.7 \\
\hline & 0.0374 & 0.0196 & 0.711 & 0.2027 & 10.34 & 113.0 \\
\hline \multirow[t]{8}{*}{313.2} & 0.0463 & 0.0013 & 0.935 & 0.0213 & 16.38 & 357.1 \\
\hline & 0.0445 & 0.0034 & 0.904 & 0.0464 & 13.65 & 262.0 \\
\hline & 0.0427 & 0.0067 & 0.858 & 0.0832 & 12.42 & 200.8 \\
\hline & 0.0406 & 0.0103 & 0.819 & 0.1140 & 11.07 & 156.8 \\
\hline & 0.0387 & 0.0139 & 0.785 & 0.1396 & 10.04 & 126.2 \\
\hline & 0.0371 & 0.0175 & 0.754 & 0.1624 & 9.280 & 104.9 \\
\hline & 0.0356 & 0.0203 & 0.738 & 0.1730 & 8.522 & 90.40 \\
\hline & 0.0343 & 0.0236 & 0.712 & 0.1927 & 8.165 & 80.72 \\
\hline \multirow[t]{8}{*}{323.2} & 0.0457 & 0.0017 & 0.932 & 0.0207 & 12.18 & 245.2 \\
\hline & 0.0439 & 0.0047 & 0.899 & 0.0468 & 9.957 & 174.8 \\
\hline & 0.0426 & 0.0094 & 0.859 & 0.0775 & 8.245 & 123.1 \\
\hline & 0.0405 & 0.0139 & 0.825 & 0.1054 & 7.583 & 103.0 \\
\hline & 0.0383 & 0.0182 & 0.797 & 0.1259 & 6.918 & 84.65 \\
\hline & 0.0360 & 0.0245 & 0.749 & 0.1619 & 6.608 & 69.68 \\
\hline & 0.0348 & 0.0269 & 0.733 & 0.1732 & 6.439 & 64.41 \\
\hline & 0.0329 & 0.0293 & 0.713 & 0.1878 & 6.410 & 60.59 \\
\hline
\end{tabular}


${ }^{\text {a }}$ Standard uncertainties $u$ are $u(T)=0.1 \mathrm{~K}, u(p)=1 \mathrm{kPa}, u(w)=0.002$ for $w>0.1, u(w)=0.0002$ for $w$ $<0.1$.

${ }^{\mathrm{b}} w_{1}$ : mass fraction of MIPK; $w_{2}$ : mass fraction of resorcinol. 


\section{Table 3}

The experimental LLE data in mass fraction for $\{$ MIPK (1) + hydroquinone (2) + water (3) $\}$ system at 298.2, 313.2 and 323.2 K under atmospheric pressure. , b $^{\text {b }}$

\begin{tabular}{|c|c|c|c|c|c|c|}
\hline \multirow[t]{2}{*}{$T / \mathrm{K}$} & \multicolumn{2}{|c|}{ Aqueous phase } & \multicolumn{2}{|c|}{ Organic phase } & \multirow[t]{2}{*}{$D$} & \multirow[t]{2}{*}{$\mathrm{S}$} \\
\hline & $w_{1}$ & $w_{2}$ & $w_{1}$ & $w_{2}$ & & \\
\hline \multirow[t]{8}{*}{298.2} & 0.0573 & 0.0017 & 0.931 & 0.0307 & 18.06 & 443.7 \\
\hline & 0.0551 & 0.0035 & 0.901 & 0.0512 & 14.63 & 288.1 \\
\hline & 0.0546 & 0.0041 & 0.890 & 0.0584 & 14.24 & 259.8 \\
\hline & 0.0529 & 0.0056 & 0.870 & 0.0764 & 13.64 & 239.6 \\
\hline & 0.0515 & 0.0069 & 0.857 & 0.0867 & 12.57 & 210.1 \\
\hline & 0.0486 & 0.0096 & 0.823 & 0.1138 & 11.85 & 176.6 \\
\hline & 0.0442 & 0.0138 & 0.783 & 0.1473 & 10.67 & 144.3 \\
\hline & 0.0380 & 0.0196 & 0.732 & 0.1864 & 9.510 & 109.8 \\
\hline \multirow[t]{8}{*}{313.2} & 0.0459 & 0.0023 & 0.926 & 0.0308 & 13.39 & 295.0 \\
\hline & 0.0445 & 0.0042 & 0.898 & 0.0529 & 12.60 & 244.0 \\
\hline & 0.0441 & 0.0052 & 0.886 & 0.0622 & 11.96 & 219.5 \\
\hline & 0.0434 & 0.0073 & 0.862 & 0.0804 & 11.01 & 181.5 \\
\hline & 0.0421 & 0.0092 & 0.845 & 0.0930 & 10.11 & 154.7 \\
\hline & 0.0413 & 0.0115 & 0.820 & 0.1121 & 9.748 & 136.0 \\
\hline & 0.0398 & 0.0139 & 0.794 & 0.1312 & 9.439 & 119.4 \\
\hline & 0.0357 & 0.0205 & 0.744 & 0.1696 & 8.273 & 90.37 \\
\hline \multirow[t]{8}{*}{323.2} & 0.0455 & 0.0028 & 0.925 & 0.0289 & 10.32 & 213.1 \\
\hline & 0.0443 & 0.0049 & 0.900 & 0.0486 & 9.918 & 183.5 \\
\hline & 0.0439 & 0.0068 & 0.881 & 0.0634 & 9.324 & 159.2 \\
\hline & 0.0431 & 0.0083 & 0.867 & 0.0719 & 8.663 & 134.5 \\
\hline & 0.0419 & 0.0101 & 0.847 & 0.0852 & 8.436 & 117.9 \\
\hline & 0.0405 & 0.0138 & 0.820 & 0.1083 & 7.848 & 103.5 \\
\hline & 0.0387 & 0.0167 & 0.797 & 0.1255 & 7.515 & 91.60 \\
\hline & 0.0366 & 0.0215 & 0.754 & 0.1533 & 7.130 & 72.45 \\
\hline
\end{tabular}


${ }^{\mathrm{a}}$ Standard uncertainties $u$ are $u(T)=0.1 \mathrm{~K}, u(p)=1 \mathrm{kPa}, u(w)=0.002$ for $w>0.1, u(w)=0.0002$ for $w$ $<0.1$.

${ }^{\mathrm{b}} w_{1}$ : mass fraction of MIPK; $w_{2}$ : mass fraction of hydroquinone. 


\section{Table 4}

Binary interaction parameters calculated from NRTL and UNIQUAC models for the ternary systems:

$\{$ MIPK (1) + (resorcinol or hydroquinone) $(2)+$ water $(3)\}$.

\begin{tabular}{|c|c|c|c|c|c|c|c|c|}
\hline \multirow[b]{2}{*}{$\mathrm{T} / \mathrm{K}$} & \multirow{2}{*}{$\begin{array}{l}\text { component } \\
\qquad i-j\end{array}$} & \multicolumn{4}{|l|}{ NRTL } & \multicolumn{3}{|c|}{ UNIQUAC } \\
\hline & & $\begin{array}{l}g_{i j}-g_{j j} \\
\left(\mathrm{~J} \cdot \mathrm{mol}^{-1}\right)\end{array}$ & $\begin{array}{l}g_{j i}-g_{i i} \\
\quad\left(\mathrm{~J} \cdot \mathrm{mol}^{-1}\right)\end{array}$ & $\alpha_{i j}$ & $\mathrm{RMSD}^{\mathrm{b}}$ & $\begin{array}{l}u_{i j}-u_{j j} \\
\left(\mathrm{~J} \cdot \mathrm{mol}^{-1}\right)\end{array}$ & $\begin{array}{cc}\mathrm{c} & u_{j i}-u_{i i} \\
& \left(\mathrm{~J} \cdot \mathrm{mol}^{-1}\right)\end{array}$ & $\operatorname{RMSD}^{\mathrm{b}}$ \\
\hline \multicolumn{9}{|c|}{ MIPK $(1)+$ resorcinol $(2)+$ water $(3)$} \\
\hline \multirow[t]{3}{*}{298.2} & $1-2$ & 1127.54 & -4482.91 & 0.3 & 0.00289 & -2480.90 & 2327.42 & 0.00235 \\
\hline & $1-3$ & 1197.22 & 10150.81 & 0.2 & & -3542.18 & -409.71 & \\
\hline & $2-3$ & -6345.33 & 14619.17 & 0.2 & & 1907.48 & -2733.98 & \\
\hline \multirow[t]{3}{*}{313.2} & $1-2$ & 1399.41 & -4639.30 & 0.3 & 0.00148 & -2419.04 & 2347.62 & 0.00165 \\
\hline & $1-3$ & 882.28 & 11459.60 & 0.2 & & -3185.01 & -704.69 & \\
\hline & $2-3$ & -6846.91 & 14970.44 & 0.2 & & 2217.34 & -3054.90 & \\
\hline \multirow[t]{3}{*}{323.2} & $1-2$ & 2940.83 & -5103.96 & 0.3 & 0.00293 & -1884.95 & 2193.15 & 0.00366 \\
\hline & $1-3$ & 634.03 & 12147.34 & 0.2 & & -2975.75 & -844.54 & \\
\hline & $2-3$ & -7067.40 & 15011.43 & 0.2 & & 2200.88 & -2584.41 & \\
\hline \multicolumn{9}{|c|}{ MIPK $(1)+$ hydroquinone $(2)+$ water $(3)$} \\
\hline \multirow[t]{3}{*}{298.2} & $1-2$ & 3920.55 & -5141.13 & 0.3 & 0.00255 & -2075.26 & 2127.64 & 0.00241 \\
\hline & $1-3$ & 1102.27 & 10215.91 & 0.2 & & -3461.53 & -427.59 & \\
\hline & $2-3$ & -6504.79 & 15322.87 & 0.2 & & 1804.80 & -2416.46 & \\
\hline \multirow[t]{3}{*}{313.2} & $1-2$ & 3926.29 & -5448.66 & 0.3 & 0.00135 & -2710.61 & 2408.73 & 0.00135 \\
\hline & $1-3$ & 1062.61 & 11265.39 & 0.2 & & -3396.93 & -636.69 & \\
\hline & $2-3$ & -7614.13 & 16791.29 & 0.2 & & 2383.79 & -3377.15 & \\
\hline \multirow[t]{3}{*}{323.2} & $1-2$ & 4169.22 & -5606.88 & 0.3 & 0.00169 & -2999.44 & 2513.24 & 0.00205 \\
\hline & $1-3$ & 920.53 & 11824.42 & 0.2 & & -3311.22 & -724.48 & \\
\hline & $2-3$ & -8231.53 & 17512.61 & 0.2 & & 2646.18 & -3756.27 & \\
\hline
\end{tabular}

${ }^{\text {a }} g_{i j}$ is interaction energy between species $i$ and $j$ in the ternary system $(\mathrm{J} / \mathrm{mol})$

b Calculated with Eq. (4)

${ }^{\mathrm{c}} u_{i j}$ is the UNIQUAC binary interaction parameter $(\mathrm{J} / \mathrm{mol})$ 


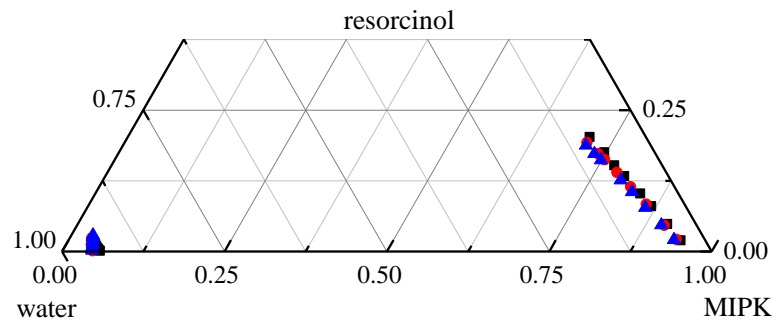

Fig. 1. LLE data for the ternary system $\{$ MIPK (1) + resorcinol (2) + water (3) $\}$ at different temperatures: $\mathbf{\square}$, at $298.2 \mathrm{~K} ; \bullet$, at $313.2 \mathrm{~K} ; \boldsymbol{\Delta}$, at $323.2 \mathrm{~K}$.

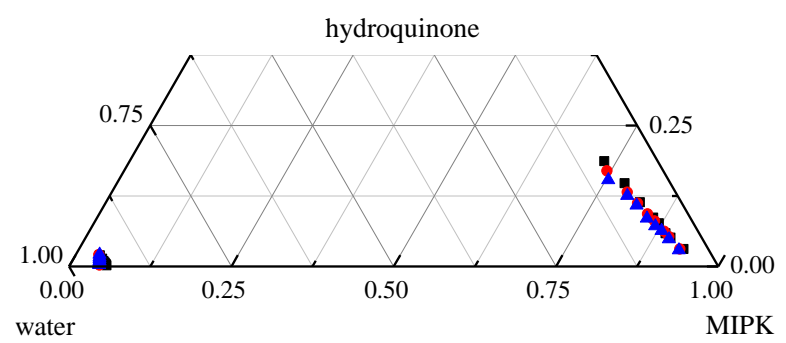

Fig. 2. LLE data for the ternary system $\{$ MIPK (1) + hydroquinone (2) + water (3) $\}$ at different temperatures: $\mathbf{\square}$, at $298.2 \mathrm{~K} ; \bullet$, at $313.2 \mathrm{~K} ; \boldsymbol{\Delta}$, at $323.2 \mathrm{~K}$. 